\title{
Efficacy and safety of omitting chest drains after video-assisted thoracoscopic surgery: a systematic review and meta-analysis
}

\author{
Lin Huang ${ }^{1}$, Henrik Kehlet ${ }^{2}$, Bo Laksáfoss Holbek ${ }^{1}$, Tina Kold Jensen ${ }^{3}$, René Horsleben Petersen ${ }^{1}$ \\ ${ }^{1}$ Department of Cardiothoracic Surgery, Copenhagen University Hospital, Rigshospitalet, Copenhagen, Denmark; ${ }^{2}$ Department of Surgical \\ Pathophysiology, Copenhagen University, Rigshospitalet, Copenhagen, Denmark; ${ }^{3}$ Department of Environmental Medicine, University of Southern \\ Denmark, Odense, Denmark \\ Contributions: (I) Conception and design: L Huang, H Kehlet, RH Petersen; (II) Administrative support: RH Petersen, H Kehlet; (III) Provision of \\ study materials: L Huang; (IV) Collection and assembly of data: L Huang, BL Holbek; (V) Data analysis and interpretation: L Huang, RH Petersen, \\ BL Holbek, TK Jensen; (VI) Manuscript writing: All authors; (VII) Final approval of manuscript: All authors. \\ Correspondence to: René Horsleben Petersen, MD, PhD. Department of Cardiothoracic Surgery, Copenhagen University Hospital, Rigshospitalet, \\ Blegdamsvej 9, 2100 Copenhagen Ø, Denmark. Email: rene.horsleben.petersen@regionh.dk.
}

Background: The aim of this systematic review and meta-analysis was to determine the efficacy and safety of omitting chest drains compared to routine chest drain placement after video-assisted thoracoscopic surgery (VATS).

Methods: Five bibliographic databases, ClinicalTrials.gov and PROSPERO were comprehensively searched from inception to July 29, 2020 (no language restrictions). Postoperative outcomes were extracted and synthesized complying with the Preferred Reporting Items for Systematic Reviews and Meta-Analyses (PRISMA). Risk of bias (RoB) including non-reporting bias, heterogeneity, and sensitivity were assessed. Subgroup analyses were performed based on study design.

Results: Of 7,166 identified studies, 10 studies [four randomized controlled trials (RCTs) and six nonRCTs] with 1,079 patients were included. There were 561 patients in the no chest drain group (NCD) and 518 patients in the standard chest drain group (CD). In pairwise analysis the NCD group had significant shorter length of stay (LOS) [weighted mean difference (WMD) -1.53 days, $\mathrm{P}<0.001$ ], less postoperative pain scores (WMD -1.09, $\mathrm{P}=0.002)$, but higher risk of drain insertion or thoracocentesis [risk radio (RR) 3.02, $\mathrm{P}=0.040]$. There were no significant differences on the incidence of minor pneumothorax ( $\mathrm{RR} 1.77$, $\mathrm{P}=0.128$ ), minor pleural effusion (RR 1.88, $\mathrm{P}=0.219)$, minor subcutaneous emphysema ( $R \mathrm{R} 1.37, \mathrm{P}=0.427$ ) or pneumonia (RR $0.53, \mathrm{P}=0.549)$. No mortality was observed in either group during the observation period (in-hospital or 30-day mortality).

Conclusions: Omitting chest drains in selected patients after VATS seems effective leading to enhanced recovery with shorter length of postoperative stay and less pain but with a higher risk of drain insertion or thoracocentesis. However, a major part of the evidence comes from observational studies with high RoB. Further RCTs are needed to improve the current evidence.

Keywords: Chest drains; video-assisted thoracoscopic surgery (VATS); meta-analysis; ERAS

Submitted Oct 22, 2020. Accepted for publication Dec 24, 2020.

doi: $10.21037 /$ jtd-20-3130

View this article at: http://dx.doi.org/10.21037/jtd-20-3130

\section{Introduction}

Video-assisted thoracoscopic surgery (VATS) has become standard of care for many thoracic procedures with favourable results on perioperative outcomes (1-4). Since the mid-1990s, when the concept of fast-track surgery or enhanced recovery after surgery (ERAS) emerged, ERAS has been introduced into the majority of surgical areas resulting in reduced length of postoperative stay (LOS), 
pain and postoperative complications $(5,6)$. In thoracic surgery, these benefits have been demonstrated in the recent publication of ERAS ${ }^{\circledR}$ guidelines (7). Chest drains are associated with pain, reduced pulmonary function, infectious complications and prolonged LOS, however with limited high-level evidence (8-10). After the shift from open thoracotomy to VATS, management of chest drains has been revised to enhance patient recovery concerning: size, removal criteria, level of suction and number of chest drains (11-15).

Due to advances in equipment and surgical technique, preliminary studies have described the feasibility of omitting chest drains after VATS resulting in shorter LOS, less pain and reduced incidence of complications $(16,17)$. However, high-level evidence from randomised controlled trials is scarce. One systematic review (18) published in 2010 including one randomized controlled trial (RCT) (19) and three observational studies (20-22), and another with meta-analysis (23) of nine observational studies (20,24-31) was published in 2018. Subsequently, additional three RCTs (32-34) and six observational studies (35-40) were published.

This updated systematic review and meta-analysis comply with the Preferred Reporting Items for Systematic Reviews and Meta-Analyses (PRISMA) guidelines (41). The aim of the study was to investigate whether intraoperative removal of chest drains leads to shorter LOS, and reduced pain, and to assess if the procedure is safe with regards to postoperative complications and short-term mortality compared to conventional treatment with a standard use of a chest drain.

We present the following article in accordance with the PRISMA reporting checklist (available at http://dx.doi. org/10.21037/jtd-20-3130).

\section{Methods}

We registered the protocol of this systematic review and meta-analysis in the International prospective register of systematic reviews (PROSPERO) database (registration number: CRD42020201291).

\section{Inclusion criteria}

* Patients aged $\geq 15$ years undergoing any VATS procedure.

* Intervention group undergoing intraoperative removal of chest drains after an air leakage test (no chest drain, NCD).
* Comparator group treated with conventional chest drain placement (CD).

- Information on at least one of the following outcomes: LOS, postoperative pain, complications and readmission.

\section{Exclusion criteria}

* Thoracotomy.

* Chest drain removal outside of operation room.

* Trial based on other intervention than intraoperative chest drain removal such as type of surgical procedure or anaesthesia.

* Letter to editor, commentary, conference abstracts, experience sharing, animal trials, case series, case reports, review and meta-analyses.

\section{Information sources and search strategy}

A systematic and comprehensive literature search was conducted via MEDLINE, EMBASE, Web of Science, and Cochrane Library from inception to July 29, 2020. We scanned the reference lists of included studies or relevant systematic reviews to identify potentially eligible studies. ClinicalTrials.gov and PROSPERO was searched for ongoing or unpublished trials and systematic reviews. The medical subjective heading term $(\mathrm{MeSH})$ and index term were used to develop detailed search strategies. If necessary, they could be modified according to requirement of variable databases. Table S1 provides full details of our search strategies.

\section{Study selection}

Initial search records were imported into Covidence (https://www.covidence.org/). The articles were screened twice based on title and abstract by one reviewer $(\mathrm{LH})$ and duplicates were excluded, and eligible full-text articles were subsequently assessed by the same reviewer. The results of aforementioned progress were checked by two reviewers (RHP and BLH). Any controversy was handled by discussion among all researchers. When multiple publications were performed on the same group of participants, the study with the highest number of participants was included.

\section{Data extraction}

A standard data extraction form was created using REDCap 
(Vanderbilt University, USA). One reviewer (LH) extracted the data twice. Another two reviewers (RHP and BLH) checked the results of data extraction. The data of interest included study characteristics (name of first author, chronology of publication, country in which the study was conducted, study design, study period), case characteristics (surgical procedures, anaesthetic method, number of ports, sample size, age, sex, method of air leakage test, eligibility criteria) and outcomes (LOS, pain, postoperative complications, readmission). In case of missing or unclear data, we contacted the corresponding author of the study for details of interpretation or additional data. Continuous variables summarised as median and interquartile range were converted to mean and standard deviation.

We recorded pain scores at postoperative day 1 (POD 1). If the study reported scores multiple times per day, the max pain score would be extracted, because this score is considered relevant for supplementary pain medication. If pain scores were reported as visual analogue scale (VAS) or numerical rating scale (NRS), data were combined. Regarding postoperative complications, we recorded pneumothorax without intervention, pleural effusion without intervention, subcutaneous emphysema without intervention, pneumonia, drain insertion or thoracocentesis (for air pneumothorax, subcutaneous emphysema or exudate), reoperation and mortality.

\section{Quality appraisal}

Two investigators (LH and RHP) independently assessed the risk of bias (RoB) for each included RCT and nonRCT respectively using RoB 2 tool and ROBINS-I tool from the Cochrane Handbook for Systematic Reviews of Interventions, then showing the results by robvis (42). A third author $(\mathrm{HK})$ acted as an arbiter when differences occurred between two investigators.

\section{Statistical analysis}

Weighted mean difference (WMD) and corresponding 95\% confidence interval $(95 \% \mathrm{CI})$ were used for continuous variables. The risk ratio (RR) with $95 \% \mathrm{CI}$ was calculated for categorical variables. The data extracted from individual studies and the results of synthesis was displayed in tables and forest plots. Heterogeneity among studies was evaluated by Cochran's $Q$ test and the $\mathrm{I}^{2}$ statistic. The values of $\mathrm{I}^{2}$ were considered high $\left(\mathrm{I}^{2}>75 \%\right)$, medium $\left(\mathrm{I}^{2}>50 \%\right)$ and low $\left(\mathrm{I}^{2}<25 \%\right)$ heterogeneity (43). A random-effect model was used if heterogeneity ( $\mathrm{Q}$ test $\mathrm{P}<0.1$ or $\mathrm{I}^{2}>50 \%$ ) was found, otherwise, a fixed-effects model was applied (43). Subgroup analyses based on study design (RCTs and non-RCTs) were conducted. In analysis, if comparable groups had zero risk ratios or relative risks undefined, the study was not included in the meta-analysis. In addition, sensitivity analyses were performed by removing each individual study in sequence from each meta-analysis group. Funnel plots were used to assess for publication bias. Then inclined funnel plots were further evaluated by Egger's test. Two-sided P values of 0.05 or less were considered statistically significant. The statistical analyses were conducted using Stata software (version 15.0, StataCorp, TX, USA).

\section{Results}

\section{Study selection}

The flowchart of study selection was shown in Figure 1. Initially, a cumulative number of 7,166 citation records were screened from MEDLINE ( $\mathrm{n}=2,267)$, EMBASE $(\mathrm{n}=2,403)$, Web of Science $(n=2,099)$ and Cochrane Library $(n=397)$. No citations were retrieved from ClinicalTrials.gov and PROSPERO. No unpublished data was found. No citations were obtained by cross-reference or related articles search. After removal of duplicates, records were screened based on inclusion and exclusion criteria on title and/or abstract. Seventeen full-text records were retrieved, of which seven records were excluded.

\section{Characteristics of studies and cases}

Ten studies with 1,079 patients were included, comprising $561(52.0 \%)$ classified as NCD and $518(48 \%)$ as CD. Among these, four (19,32-34) were RCTs including 353 patients $(33 \%)$ and six $(27-30,38,39)$ were non-RCTs including 726 patients (all retrospective but one). The number of patients for each individual study ranged from 49 to 333. None of the studies included anatomical resections. Details of included studies is summarised in Table 1.

Additionally, duration of chest drainage in the CD group was reported by eight studies (27-29,32-34,38,39), of which range of mean was from 1 day to 4.23 days.

\section{Quality assessment}

Overall, 30\% included studies were moderate RoB and $70 \%$ were high risk. The graph and details of RoB for included 


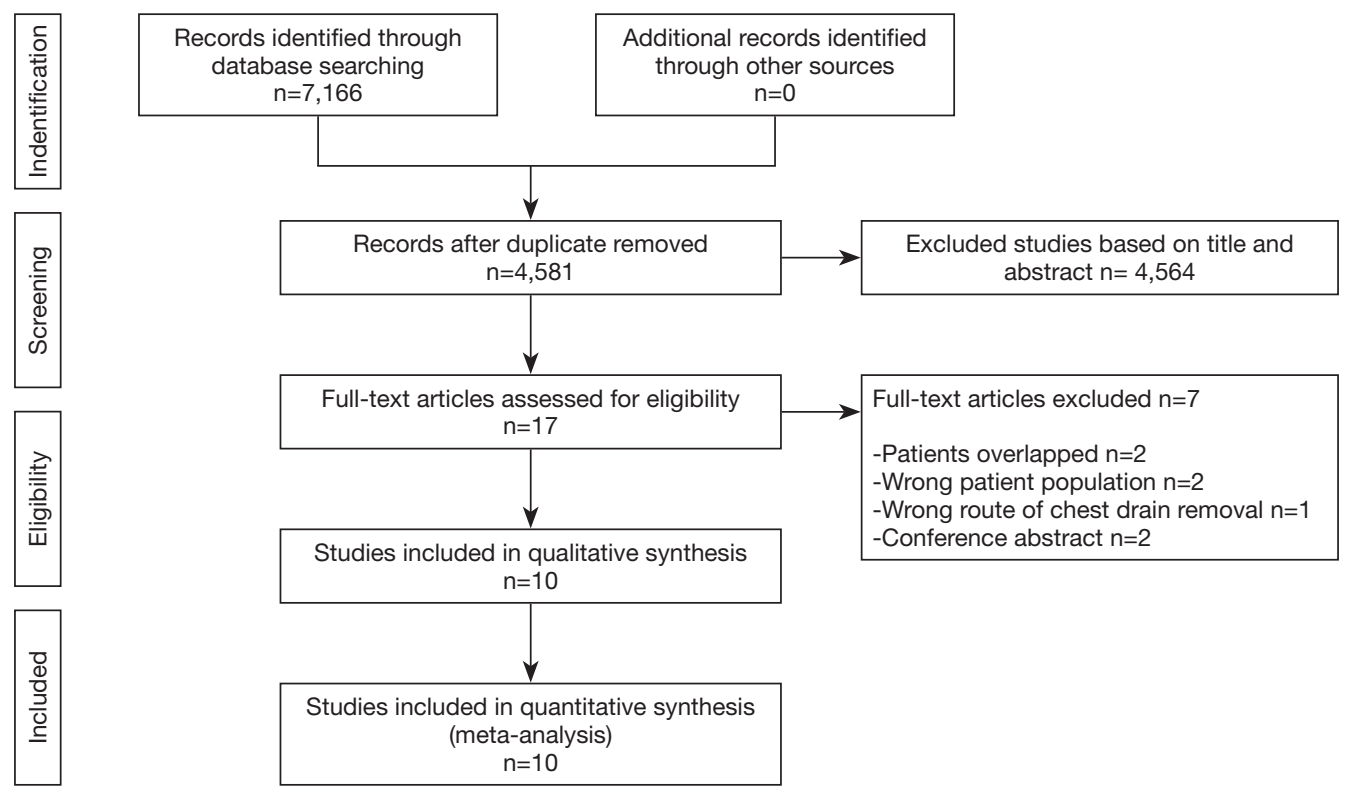

Figure 1 The Preferred Reporting Item for Systematic Reviews and Meta-Analysis (PRISMA) flowchart of selection procedure for the studies.

Table 1 Basic characteristics of included studies

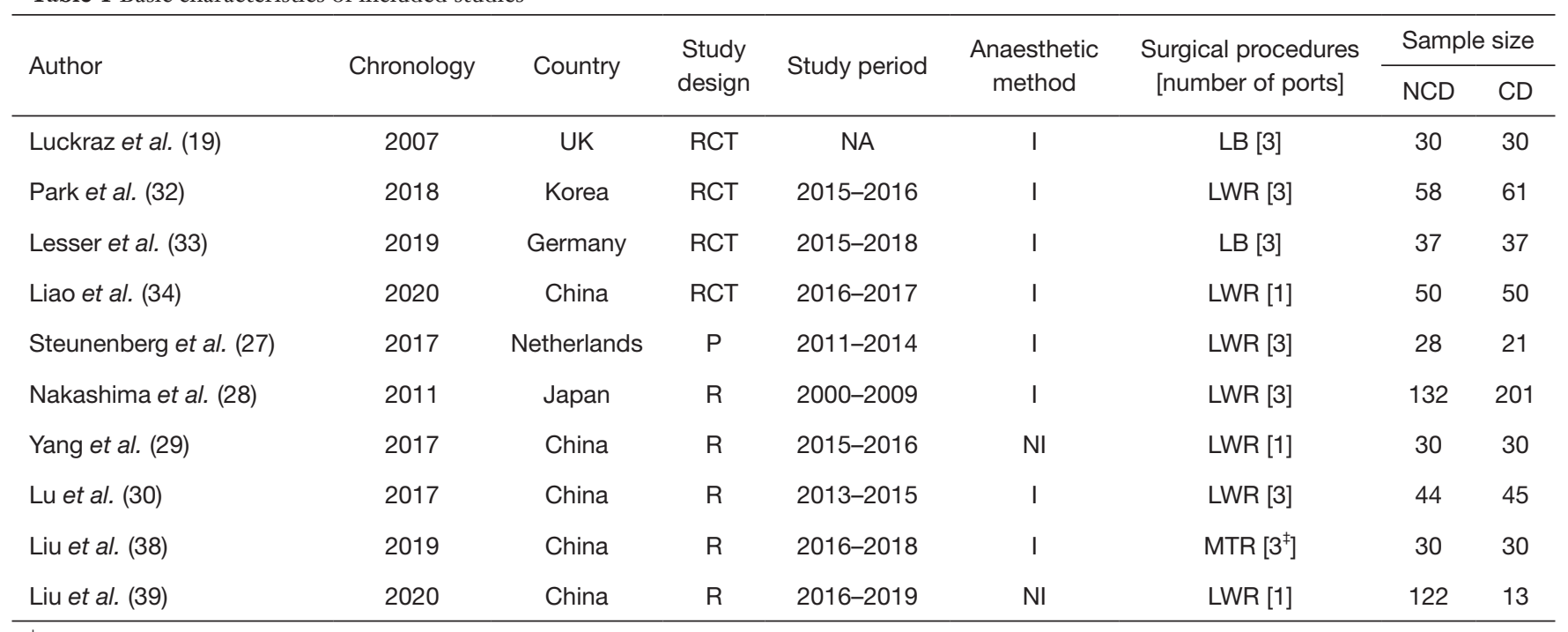

${ }^{\ddagger}$, most patients received three-ports thoracoscopic surgery while selective patients received reduced-port technique. CD, chest drain group; I, intubated anaesthesia; LB, lung biopsy; LWR, lung wedge resection; MTR, mediastinal tumour resection; NCD, no chest drain group; NI, nonintubated anaesthesia; NA, not available; P, prospective study; R, retrospective study; RCT, randomized controlled trial; UK, United Kingdom.

studies is shown in Figure 2.

\section{Outcomes}

All included studies reported LOS (Table 2). The overall result showed that individuals in the NCD group had
1.53 days shorter LOS than that in the CD group. RCT subgroup analysis showed patients with NCD had 1.59 shorter LOS and non-RCTs subgroup analysis showed patients with CD had 1.48 days shorter LOS. Overall and subtotal analyses showed medium heterogeneity, and heterogeneity of the RCT subgroup analysis was low 

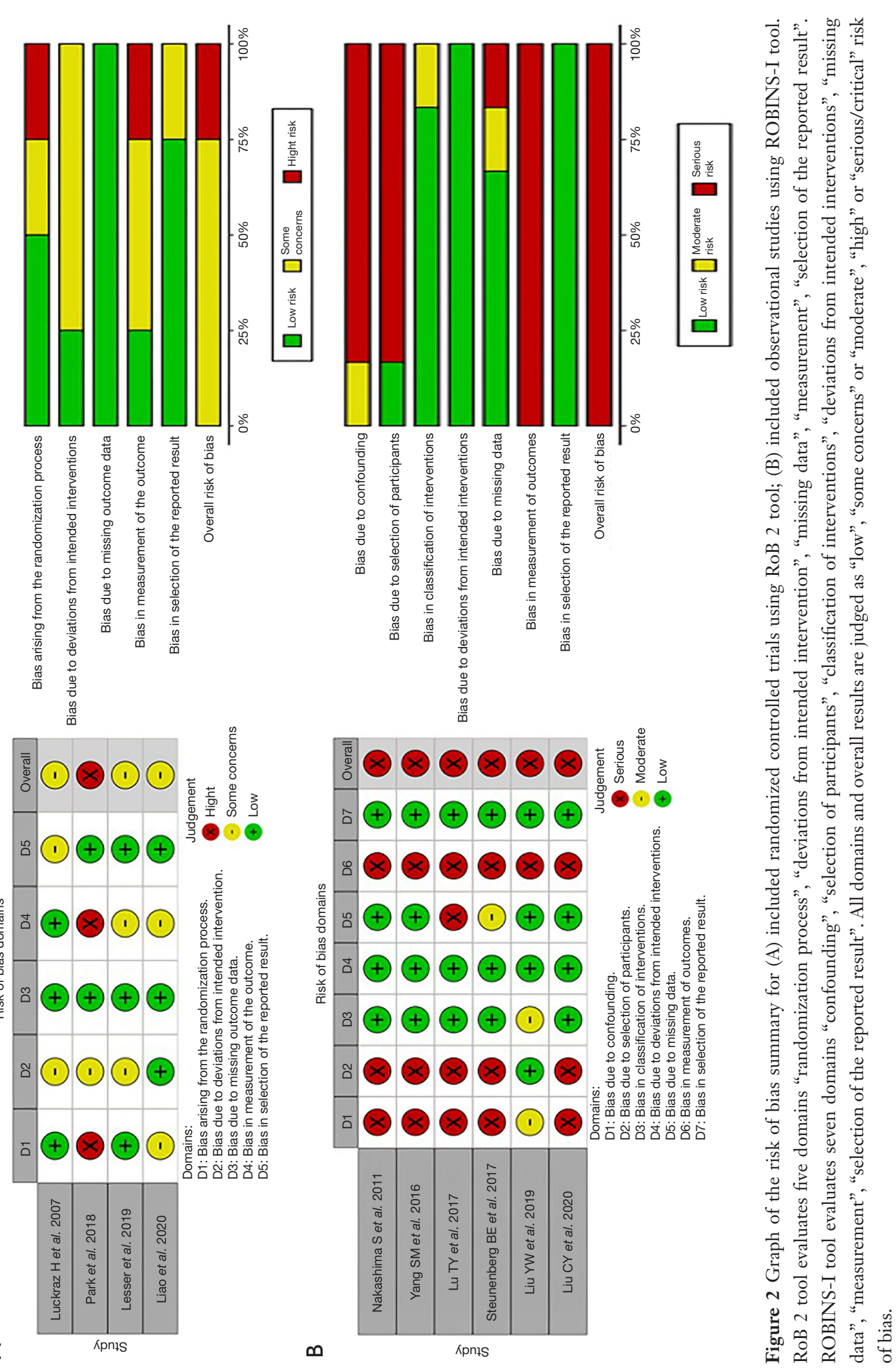
Table 2 Main outcomes of included studies

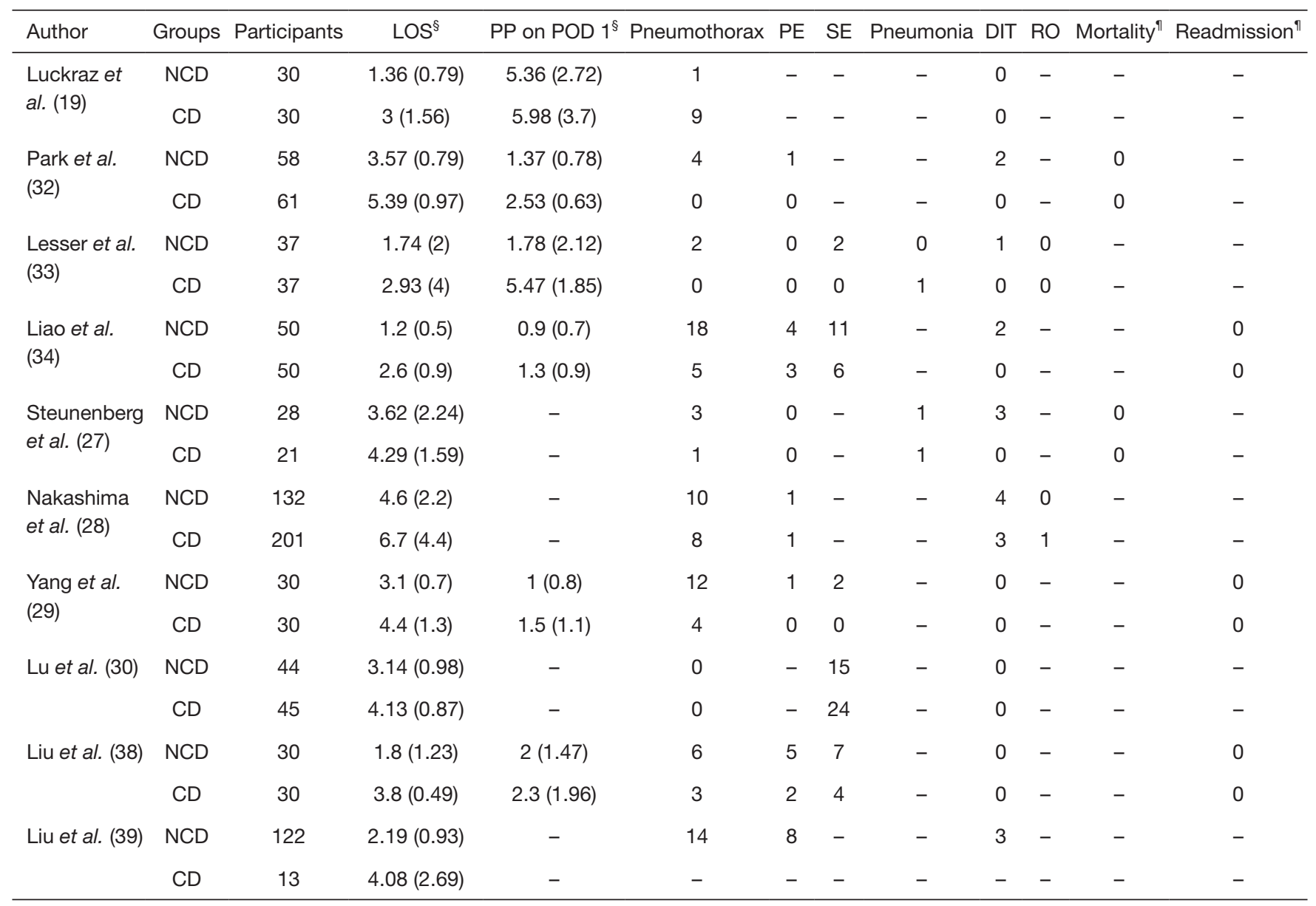

-, no reported data; ${ }^{\S}$, data presented as mean (standard deviation); ", in 30 days after surgery. CD, chest drain group; LOS, length of postoperative stay; NCD, no chest drain group; PE, pleural effusion; POD1, postoperative day 1; PP, postoperative pain; DIT, drain insertion or thoracocentesis; $\mathrm{RO}$, reoperation; $\mathrm{SE}$, subcutaneous emphysema.

\section{(Table 3, Figure 3).}

Of the ten included studies, six reported data on postoperative pain by VAS or NRS with eleven points, comprising four RCTs (19,32-34) and two non-RCTs $(30,38)$ (Table 2) with 249 patients in the NCD-group and 253 patients in the CD-group. Patients with NCD had 1.09 lower pain scores than CD in overall result. In RCT and non-RCT subgroup analysis, patient in the NCD group had respectively 1.46 and 0.45 lower pain scores than in the $\mathrm{CD}$ group. There was high heterogeneity in the overall analysis and RCT subgroup analysis; non-RCT subgroup analysis did not show heterogeneity (Table 3, Figure 4).

Eight studies reporting postoperative pneumothorax, which did not require drain insertion or thoracocentesis was included in the analysis, comprising four RCTs (19,32-34) and four non-RCTs $(27-29,38)$ (Table 2). There were 395 patients with NCD and 460 with CD. The difference between the NCD group and CD group was not statistically significant, except the result of non-RCT subgroup showed patients in the NCD group with high risk. The heterogeneity of overall analysis was close to medium, as well as the RCT subgroup analysis showed medium heterogeneity. Non-RCT subgroup analysis did not show heterogeneity (Table 3, Figure 5).

Eight studies reported postoperative pleural effusion without the need for drainage or thoracocentesis (27-29,32-34,38,39). From four studies (one was RCT and two were non-RCTs) comprising each 110 patients in both the NCD group and the CD group $(29,34,38)$, with no significant difference between the two groups and not difference on subgroup analysis. There was no heterogeneity among analyses (Table 3, Figure 5). 
Table 3 Outcomes of meta-analysis

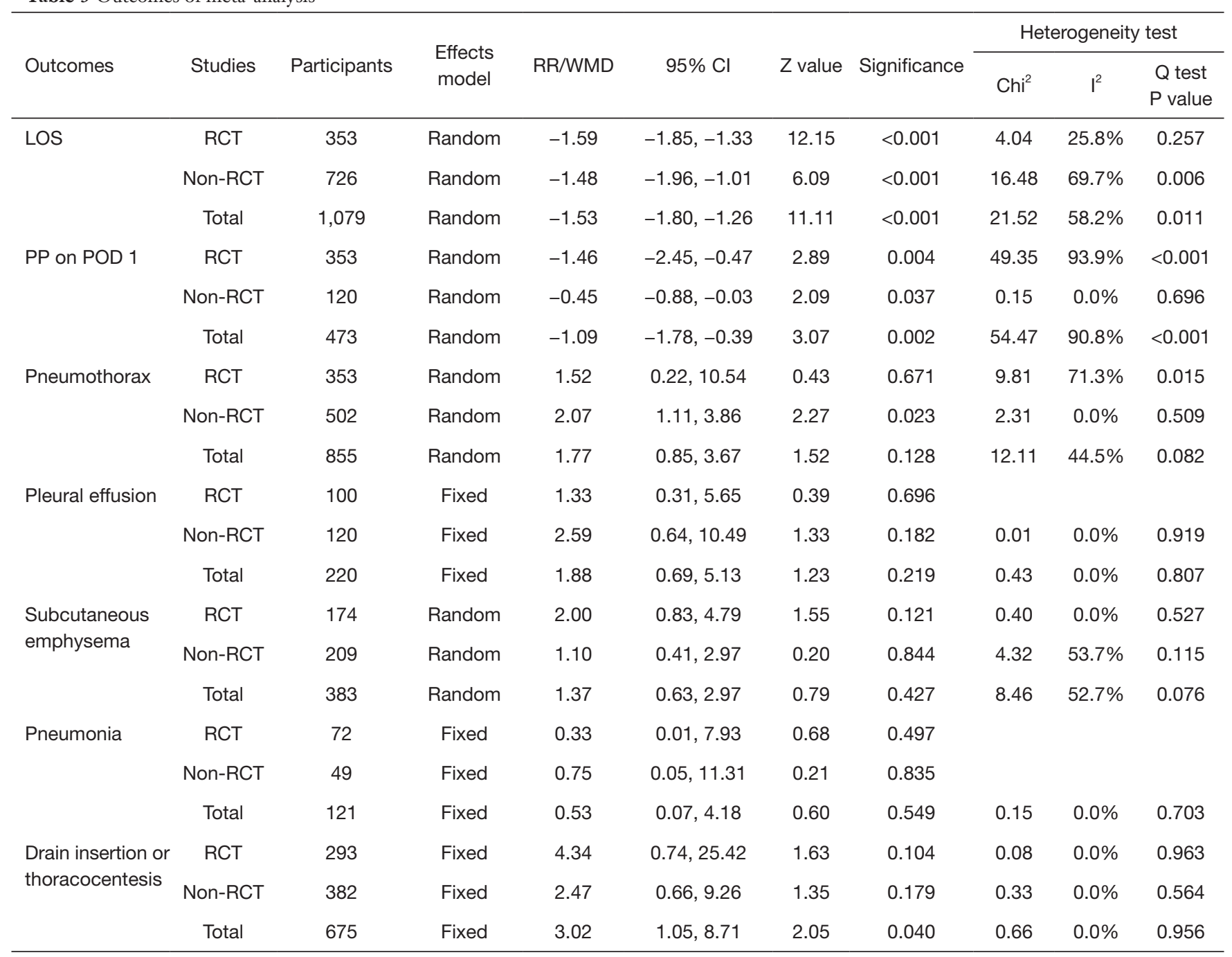

$\mathrm{Cl}$, confidence intervals; LOS, length of postoperative stay; non-RCT, no randomized controlled trial; POD1, postoperative day 1; PP, postoperative pain; $\mathrm{RCT}$, randomized controlled trial; RR, risk ratio; WMD, weighted mean difference.

Data of subcutaneous emphysema was extracted from two RCTs $(33,34)$ and three non-RCTs $(29,30,38)$, which comprised 191 cases in the NCD group and 192 cases in the $\mathrm{CD}$ group. The difference between the two groups was not significant in overall and subtotal analyses. There was medium heterogeneity in the overall analysis and non-RCT subgroup analysis; no heterogeneity was found in the RCT subgroup analysis (Table 3, Figure 5).

Only two studies (one RCT and one non-RCT) reported data on pneumonia rate, which comprised 65 patients with NCD and 58 with CD $(27,33)$, the difference between two groups was not significant, and with no indications of heterogeneity (Table 3, Figure 5).
Five studies reporting data about drain insertion or thoracentesis were synthesized and analysed (27,28,32-34) comprising 305 patients with NCD and 370 with CD. All the procedures were performed during the in-hospital stay. The NCD group had higher risk of drain insertion or thoracentesis $(3.93 \%)$ than the CD group $(0.81 \%)$ in overall analysis but not in either subgroup analysis, which did not show heterogeneity (Table 3, Figure 5). Data of reoperation was extracted from two studies, of which only one showed a patient who was reoperated because of bleeding (28) (Table 3).

Follow up was very variable in the included studies. One study had only seven days follow up (33). One study had 


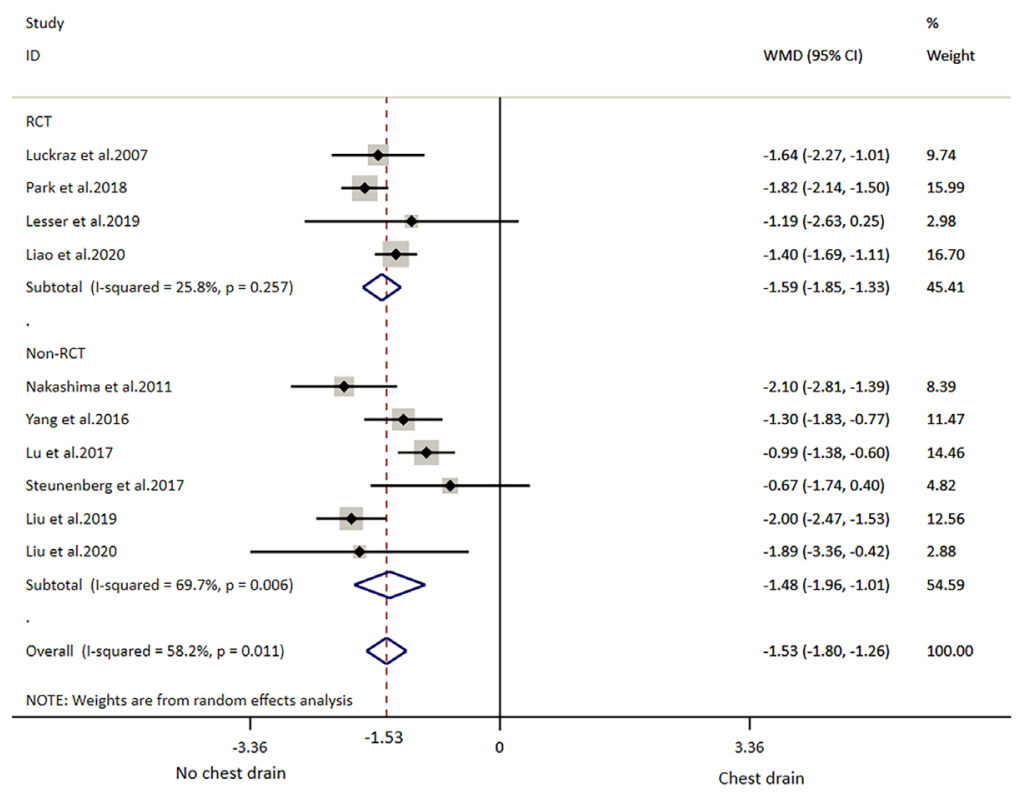

Figure 3 Meta-analysis of length of postoperative stay (LOS) and subgroup analyses. WMD, weighted mean deviation; CI, confidence intervals. The red dotted line indicates the overall result.

only 2 weeks follow up (39). Six studies had one month follow up (19,27-30,38) and the remaining two studies followed up 6 to 12 months $(32,34)$.

The 30-day mortality after surgery was reported in two studies, without mortality in any group (Table 3).

Readmission in 30-day after surgery was reported in three studies, without readmission in any group (Table 3).

\section{Sensitivity analysis and publication bias}

Sensitivity analyses showed that all studies could be included for analysis. Funnel plots did not show any publication bias (Figure S1). Simultaneously, Egger' s test was further testified for asymmetry funnel plots without significant bias $(\mathrm{P}>0.05)$.

\section{Discussion}

Management of chest drain is a critical component of an enhanced recovery pathway (6). In this updated systematic review, five additional studies ( 3 RCTs and 2 non-RCTs) were analysed compared to a previous review from 2018 (32-34,38,39). This updated meta-analysis includes variable thoracic surgical procedures performed with VATS. It is highlighted that the current study explored the effect of postoperative complications on patients after VATS in depth. Despite an increasing number of publications demonstrating the beneficial outcomes by omitting chest drain after thoracic surgery, few studies focused on adverse events with NCD.

Omitting chest drain after VATS has only been applied in selective patients. Based on reviewing the included literature combined with our own practice, patient selection is mainly separated into two parts: baseline characteristics and intraoperative air leakage test (Table S2).

Omitting chest drain contributed to shorter LOS ranging from 0.99 day to 2.10 days among included studies. As for heterogeneity in overall and subtotal analyses, we find that study design and surgical procedure are limiting factors for the conclusion.

Different criteria for removal of chest drains may lead to different duration of drainage. For instance, few institutions applied removal of chest drain within 24 after surgery, so we decided to record and analyse pain scores on POD 1. Overall, patients had less pain on POD 1 in the NCD group than in the CD group among all relevant studies. According to our analyses, high heterogeneity was associated to different study design.

As for postoperative pneumothorax, NCD was not associated to an increased risk based on results of the 


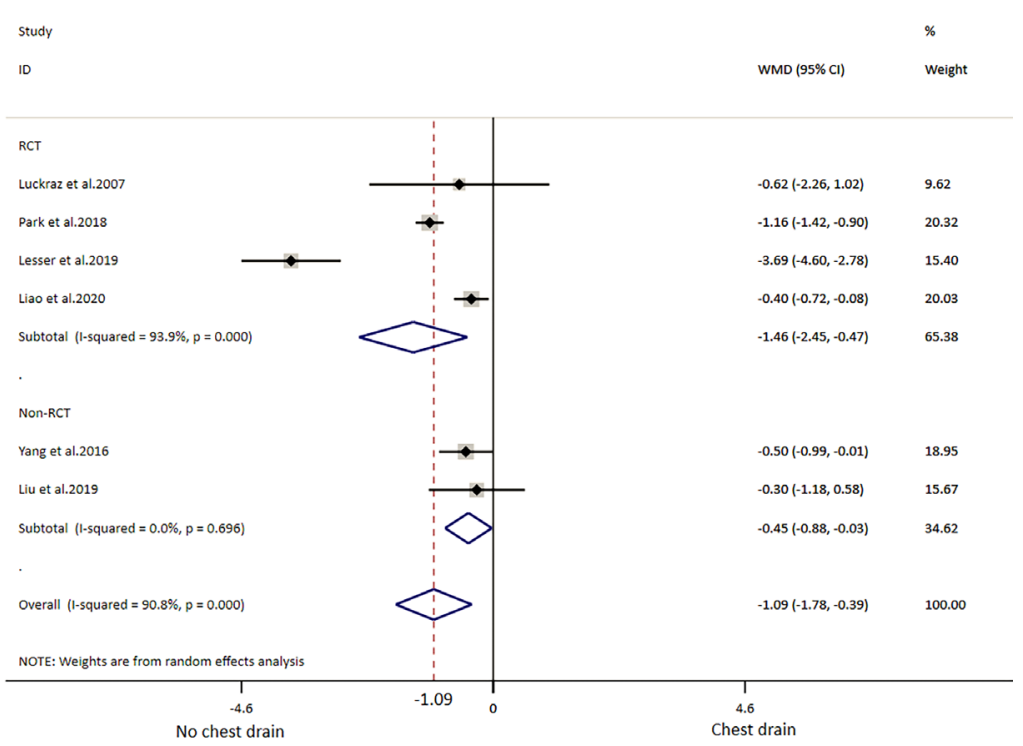

Figure 4 Meta-analysis of pain scores. WMD, weighted mean deviation; CI, confidence intervals. The red dotted line indicates the overall result.

overall and RCT subgroup analyses. However, regarding other postoperative complications, NCD increased drain insertion or thoracocentesis. Recently, Liu et al. (39) reported drain insertion or thoracocentesis in the NCD group $(0.8 \%$ because of pneumothorax and $1.6 \%$ because of pleural effusion), but this was not included in our analysis. According to our synthesis, 3.28\% drain insertion or thoracocentesis was for pneumothorax and $0.65 \%$ for pleural effusion in the NCD group, while $0.54 \%$ drain insertion or thoracocentesis was for pneumothorax and $0.27 \%$ for pleural effusion in the CD group. We consider that prolonged air leakage or unexpected pleural effusion could be drained quickly for patients through chest drain while patients with NCD have to absorb slowly by themselves. Requirement of drain insertion or thoracocentesis did not affect the overall difference in patient discharge between groups, however for the single patient the LOS was prolonged. Meanwhile, it is critical that surgeons are capable to cure severe pneumothorax or pleural effusion once occurring. The risk of not discovering a massive haemorrhage is a concern, when omitting a chest drain and careful selection of patients and postoperative monitoring including additional chest $\mathrm{X}$-ray is advisable.

The present updated systematic review and meta-analysis has limitations. First, due to the small number of included studies, covering a minority of high-quality studies, RCTs and non-RCTs were synthetically analysed and may be influenced by potential selection and reporting bias. Also, confounding by indications cannot be excluded in nonRCTs. Several included studies could not be included in the meta-analyse because both groups had event rates of zero odds ratios and relative risks undefined, which further increased the rate of bias. In addition, parts of the subgroups prevented enough power, as some results of the meta-analysis were defined by a single study. Furthermore, interquartile range converted into mean and standard deviation may result in statistical bias. Finally, included studies lacking major surgery, for example, lobectomy and segmentectomy, might add to bias.

Other factors such as the type and size of a chest drains, additional anesthesia (epidural or inter-costal block) may impact the pain scores.

The strength of this study is the up-to-date analysis with detailed literature search.

\section{Conclusions}

Omitting chest drain in selected patients undergoing VATS procedures may be effective leading to shorter LOS and less pain with no observed increase in minor complications, reoperation, mortality or readmission, except increasing drain insertion or thoracocentesis. However, majority of evidence is not high quality, so larger well-designed prospective RCT's are required to strengthen the current evidence. 


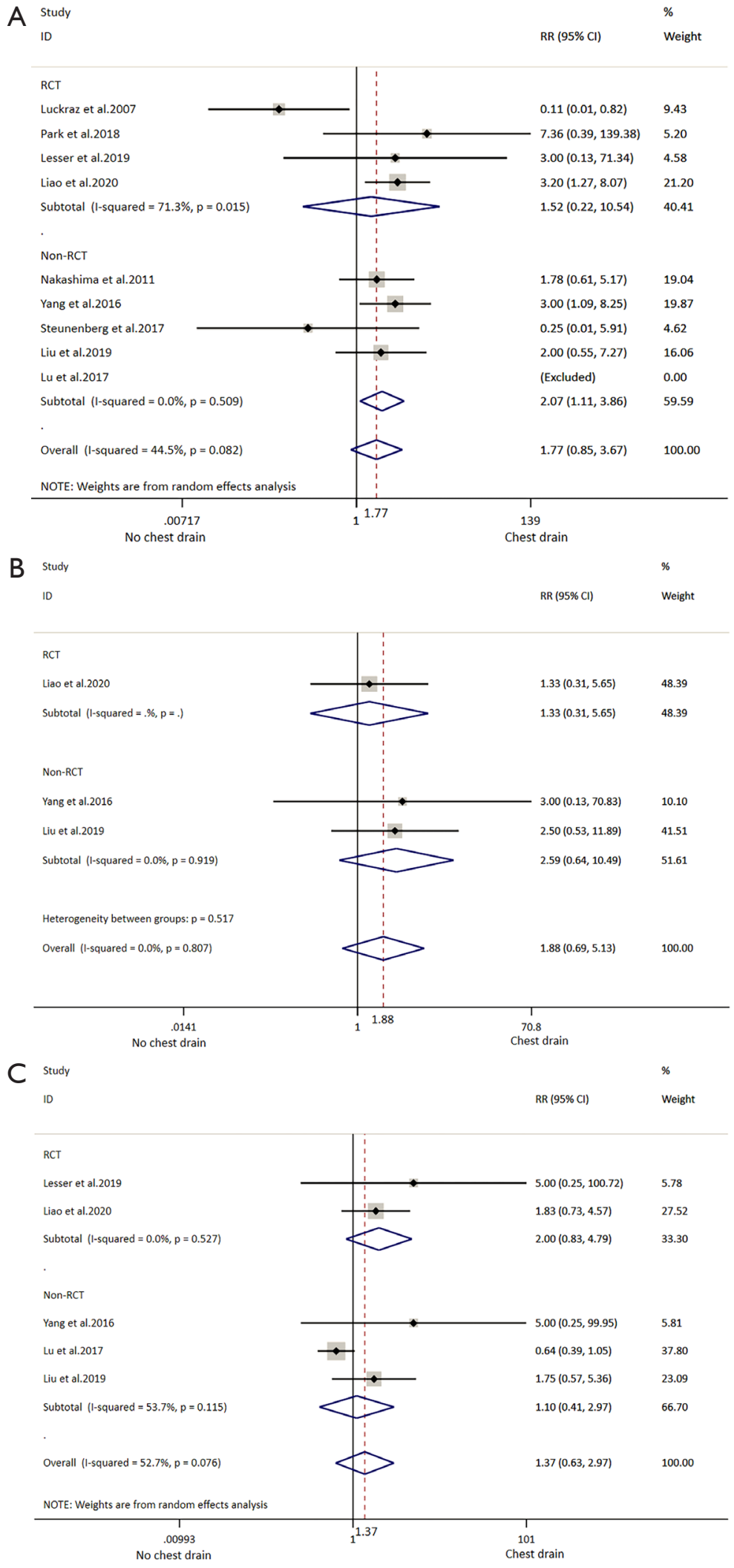




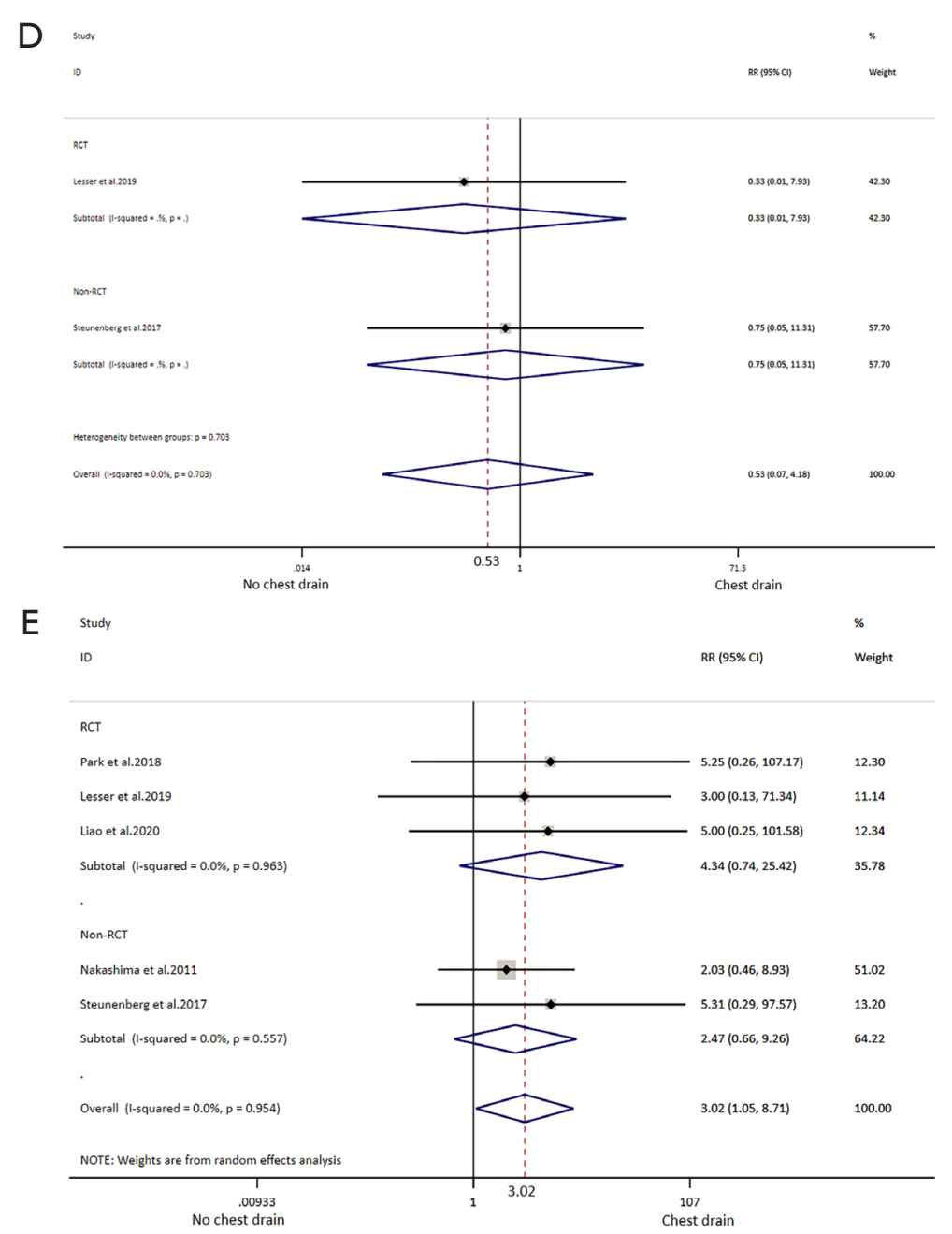

Figure 5 Meta-analysis and subgroup analyses of (A) pneumothorax; (B) pleural effusion; (C) subcutaneous emphysema; (D) pneumonia; (E) drain insertion or thoracentesis. RR, risk ratio; CI, confidence intervals. The red dotted line indicates the overall result.

\section{Acknowledgments}

Meeting presentation: an abstract for the study was presented as a poster presentation at the 1 st virtual and 28th European Conference on General Thoracic Surgery (ESTS), 2nd-3rd October 2020.

Funding: LH was financially supported by China Scholarship Council (No. 201908430204).

\section{Footnote}

Reporting Checklist: The authors have completed the PRISMA reporting checklist. Available at http://dx.doi. org/10.21037/jtd-20-3130
Conflicts of Interest: All authors have completed the ICMJE uniform disclosure form (available at http://dx.doi. org/10.21037/jtd-20-3130). RH Petersen reports personal fees from Medtronic, personal fees from Astra Zeneca, outside the submitted work. RHP serves as an unpaid editorial board member of Fournal of Thoracic Disease from Sep 2020 to Aug 2022. BLH reports a speaker's fee from Medela Healthcare, outside the submitted work. The other authors have no conflicts of interest to declare.

Ethical Statement: The authors are accountable for all aspects of the work in ensuring that questions related to the accuracy or integrity of any part of the work are appropriately investigated and resolved. 
Open Access Statement: This is an Open Access article distributed in accordance with the Creative Commons Attribution-NonCommercial-NoDerivs 4.0 International License (CC BY-NC-ND 4.0), which permits the noncommercial replication and distribution of the article with the strict proviso that no changes or edits are made and the original work is properly cited (including links to both the formal publication through the relevant DOI and the license). See: https://creativecommons.org/licenses/by-nc-nd/4.0/.

\section{References}

1. Jawitz OK, Wang Z, Boffa DJ, et al. The differential impact of preoperative comorbidity on perioperative outcomes following thoracoscopic and open lobectomies. Eur J Cardiothorac Surg 2017;51:169-74.

2. Yan TD, Black D, Bannon PG, et al. Systematic review and meta-analysis of randomized and nonrandomized trials on safety and efficacy of video-assisted thoracic surgery lobectomy for early-stage non-small-cell lung cancer. J Clin Oncol 2009;27:2553-62.

3. Nagpal K, Ahmed K, Vats A, et al. Is minimally invasive surgery beneficial in the management of esophageal cancer? A meta-analysis. Surg Endosc 2010;24:1621-9.

4. Friedant AJ, Handorf EA, Su S, et al. Minimally Invasive versus Open Thymectomy for Thymic Malignancies: Systematic Review and Meta-Analysis. J Thorac Oncol 2016;11:30-8.

5. Kehlet H. Multimodal approach to control postoperative pathophysiology and rehabilitation. Br J Anaesth 1997;78:606-17.

6. Kehlet H, Wilmore DW. Evidence-based surgical care and the evolution of fast-track surgery. Ann Surg 2008;248:189-98.

7. Batchelor TJ, Rasburn NJ, Abdelnour-Berchtold E, et al. Guidelines for enhanced recovery after lung surgery: recommendations of the Enhanced Recovery After Surgery (ERAS(R)) Society and the European Society of Thoracic Surgeons (ESTS). Eur J Cardiothorac Surg 2019;55:91-115.

8. Mao M, Hughes R, Papadimos TJ, et al. Complications of chest tubes: a focused clinical synopsis. Curr Opin Pulm Med 2015;21:376-86.

9. Mueller XM, Tinguely F, Tevaearai HT, et al. Impact of duration of chest tube drainage on pain after cardiac surgery. Eur J Cardiothorac Surg 2000;18:570-4.

10. Refai M, Brunelli A, Salati M, et al. The impact of chest tube removal on pain and pulmonary function after pulmonary resection. Eur J Cardiothorac Surg 2012;41:820-2; discussion 823.

11. Holbek BL, Christensen M, Hansen HJ, et al. The effects of low suction on digital drainage devices after lobectomy using video-assisted thoracoscopic surgery: a randomized controlled trial. Eur J Cardiothorac Surg 2019;55:673-81.

12. Bertholet JW, Joosten JJ, Keemers-Gels ME, et al. Chest tube management following pulmonary lobectomy: change of protocol results in fewer air leaks. Interact Cardiovasc Thorac Surg 2011;12:28-31.

13. Lijkendijk M, Licht PB, Neckelmann K. Electronic versus traditional chest tube drainage following lobectomy: A randomized trial. Eur J Cardiothorac Surg 2015;48:893-8.

14. Rahman NM, Pepperell J, Rehal S, et al. Effect of opioids vs NSAIDs and larger vs smaller chest tube size on pain control and pleurodesis efficacy among patients with malignant pleural effusion: The TIME1 Randomized Clinical Trial. JAMA 2015;314:2641-53.

15. Brunelli A, Beretta E, Cassivi SD, et al. Consensus definitions to promoter an evidence-based approach to management of the pleural space. A collaborative proposal by ESTS, AATS, STS, and GTSC. Eur J Cardiothorac Surg 2011;40:291-7.

16. Holbek BL, Hansen HJ, Kehlet H, et al. Thoracoscopic pulmonary wedge resection without post-operative chest drain: an observational study. Gen Thorac Cardiovasc Surg 2016;64:612-7.

17. Ueda K, Haruki T, Murakami J, et al. No Drain After Thoracoscopic Major Lung Resection for Cancer Helps Preserve the Physical Function. Ann Thorac Surg 2019;108:399-404.

18. Koç T, Routledge T, Chambers A, et al. Do patients undergoing lung biopsy need a postoperative chest drain at all? Interact Cardiovasc Thorac Surg 2010;10:1022-5.

19. Luckraz H, Rammohan KS, Phillips M, et al. Is an intercostal chest drain necessary after video-assisted thoracoscopic (VATS) lung biopsy? Ann Thorac Surg 2007;84:237-9.

20. Watanabe A, Watanabe T, Ohsawa H, et al. Avoiding chest tube placement after video-assisted thoracoscopic wedge resection of the lung. Eur J Cardiothorac Surg 2004;25:872-6.

21. Ponsky TA, Rothenberg SS, Tsao K, et al. Thoracoscopy in children: is a chest tube necessary? J Laparoendosc $\mathrm{Adv}$ Surg Tech A 2009;19:S23-S25

22. Satherley LK, Luckraz H, Rammohan KS, et al. Routine placement of an intercostal chest drain during videoassisted thoracoscopic surgical lung biopsy unnecessarily 
prolongs in-hospital length of stay in selected patients. Eur J Cardiothorac Surg 2009;36:737-40.

23. Li P, Shen C, Wu Y, et al. It is safe and feasible to omit the chest tube postoperatively for selected patients receiving thoracoscopic pulmonary resection: a meta-analysis. J Thorac Dis 2018;10:2712-21.

24. Murakami J, Ueda K, Tanaka T, et al. The Validation of a No-Drain Policy After Thoracoscopic Major Lung Resection. Ann Thorac Surg 2017;104:1005-11.

25. Ueda K, Hayashi M, Tanaka T, et al. Omitting chest tube drainage after thoracoscopic major lung resection. Eur J Cardiothorac Surg 2013;44:225-9.

26. Cui F, Liu J, Li S, et al. Tubeless video-assisted thoracoscopic surgery (VATS) under non-intubated, intravenous anesthesia with spontaneous ventilation and no placement of chest tube postoperatively. J Thorac Dis 2016;8:2226-32.

27. Steunenberg BE., Aerts BAC, de Groot HGW, et al. Is it still necessary to leave a chest tube after video-assisted pulmonary wedge resection? Indian J Thorac Cardiovasc Surg 2017;33:132-6.

28. Nakashima S, Watanabe A, Mishina T, et al. Feasibility and safety of postoperative management without chest tube placement after thoracoscopic wedge resection of the lung. Surg Today 2011;41:774-9.

29. Yang SM, Wang ML, Hung MH, et al. Tubeless Uniportal Thoracoscopic Wedge Resection for Peripheral Lung Nodules. Ann Thorac Surg 2017;103:462-8.

30. Lu TY, Chen JX, Chen PR, et al. Evaluation of the necessity for chest drain placement following thoracoscopic wedge resection. Surg Today 2017;47:606-10.

31. Russo L, Wiechmann RJ, Magovern JA, et al. Early chest tube removal after video-assisted thoracoscopic wedge resection of the lung. Ann Thorac Surg 1998;66:1751-4.

32. Park JB, Hwang JJ, Lee WS, et al. Postoperative chest tube placement after thoracoscopic wedge resection of lung for primary spontaneous pneumothorax: is it mandatory? J

Cite this article as: Huang L, Kehlet H, Holbek BL, Jensen TK, Petersen RH. Efficacy and safety of omitting chest drains after video-assisted thoracoscopic surgery: a systematic review and meta-analysis. J Thorac Dis 2021;13(2):1130-1142. doi: $10.21037 /$ jtd-20-3130
Thorac Dis 2018;10:4812-8.

33. Lesser T, Doenst T, Lehmann T, et al. Lung Bioposy Without Pleural Drainage. Dtsch Arztebl Int 2019;116:329-34.

34. Liao HC, Yang SM, Hung MH, et al. Thoracoscopic Surgery Without Drainage Tube Placement for Peripheral Lung Nodules. Ann Thorac Surg 2020;109:887-93.

35. Li S, Jiang L, Ang KL, et al. New tubeless video-assisted thoracoscopic surgery for small pulmonary nodules. Eur J Cardiothorac Surg 2017;51:689-93.

36. Liu CY, Hsu PK, Chien HC, et al. Tubeless single-port thoracoscopic sublobar resection: indication and safety. J Thorac Dis 2018;10:3729-37.

37. Xu H, Guo C, Li Y, et al. Omitting Chest Tube Drainage after Subxiphoid Thoracoscopic Thymectomy. Thorac Cardiovasc Surg 2020;68:446-9.

38. Liu YW, Chen HW, Lee JY, et al. Is a Chest Tube Necessary after Video-Assisted Thoracoscopic Mediastinal Tumor Resection? Thorac Cardiovasc Surg 2019. doi: 10.1055/s-0039-1683879.

39. Liu CY, Hsu PK, Leong KI, et al. Is tubeless uniportal video-assisted thoracic surgery for pulmonary wedge resection a safe procedure? Eur J Cardiothorac Surg 2020;58:i70-i76.

40. Li J, Qi G, Zhang X, et al. Is chest tube drainage necessary after subxiphoid thoracoscopic thymectomy? J Cardiothorac Surg 2020;15:66.

41. Moher D, Liberati A, Tetzlaff J, et al. Preferred reporting items for systematic reviews and meta-analysis: the PRISMA statement. BMJ 2009;339:b2535.

42. Higgins JPT, Thomas J, Chandler J, et al. Cochrane Handbook for Systematic Reviews of Interventions version 6.0 (updated July 2019). Cochrane, 2019. Available online: www.training.cochrane.org/handbook

43. Higgins JPT, Thompson SG, Deeks JJ, et al. Measuring inconsistency in meta-analyses. BMJ 2003;327:557-60. 
\#3 VATS[MeSH Terms],,," "' "thoracic surgery, video-assisted" "[MeSH Terms"

\#4 VATS[THle/ABstract,,", VATS" [Title/Abstract "

\#6 thoracoscopic surgery[Title/Abstract]],," " " thoracoscopic surgery" "[Title/Abstract] "

\#7 thoracoscope[MeSH Terms],,," " "thoracoscopes" "[MeSH Terms]"

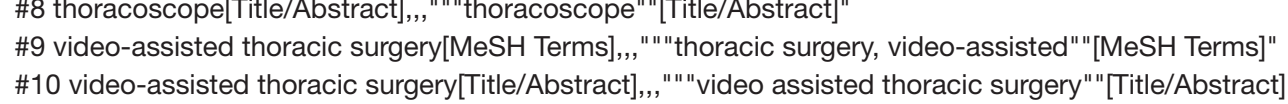

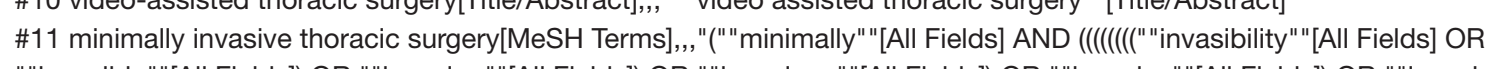

" "invasible" "[All Fields]) OR " "invasion" "[All Fields]) OR " "invasions" "[All Fields]) OR " "invasive" "[All Fields]) OR " "invasively" "[All Fields]) OR " "invasiveness" "[All Fields]) OR " "invasives" "[All Fields]) OR " "invasivity" "[All Fields])) AND (" "thoracic surgical procedures" "[MeSH Terms] OR " "thoracic surgery" "[MeSH Terms])"

\#12 minimally invasive thoracic surgery[Title/Abstract]],," " "minimally invasive thoracic surgery" "[Title/Abstract]"

\#13 thoracoscopy[MeSH Terms]],," " "thoracoscopy" "[MeSH Terms]"

\#14 thoracoscopy[Title/Abstract]],," " "thoracoscopy" "[Title/Abstract]"

\#15 (\#1 OR \#2 OR \#3 OR \#4 OR \#5 OR \#6 OR \#7 OR \#8 OR \#9 OR \#10 OR \#11 OR \#12 OR \#13 OR \#14)

\#16 chest tube[MeSH Terms],,," " "chest tubes" "[MeSH Terms]'

\#17 chest tube[Title/Abstract],,," " "chest tube" "[Title/Abstract]"

\#18 chest drain[MeSH Terms],,", " "chest tubes" "[MeSH Terms]

\#19 chest drain[Title/Abstract],,," " "chest drain" "[Title/Abstract]",

\#20 chest drainage[MeSH Terms],,,"((/" "chested" "[All Fields] OR " "thorax" "[MeSH Terms]) OR " "thorax" "[All Fields]) OR

" "chest" "[All Fields]) OR " "chests" "[All Fields]) AND " "drainage" "[MeSH Terms]

\#21 chest drainage[Titte/Abstract]],,", "'chest drainage" "[Title/Abstract]"

\#22 pleural drainage[MeSH Terms],,,"(" "pleura" "[MeSH Terms] OR ""pleura" "[All Fields]) OR " "pleural" "[All Fields]) AND "'drainage" "[MeSH Terms]"

\#23 pleural drainage[Title/Abstract],,, " " "pleural drainage" " [Title/Abstract]"

\#24 thorax drainage[MeSH Terms],,,"(" "thorax" "[MeSH Terms] OR ""thorax" "[All Fields]) OR " "thoraxes" "[All Fields]) AND "'"drainage" "[MeSH Terms]"

(T)

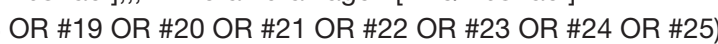

\#27 (\#15 AND \#26)

EMBASE \#1 video-assisted thoracoscopic surgery.ti. or video-assisted thoracoscopic surgery.ab. or video assisted thoracoscopic surgery.ti. or video assisted thoracoscopic surgery.ab. or thoracic surgery, video-assisted.ti. or thoracic surgery, video-assisted. ab. or VATS.ti. or VATS.ab. or thoracoscopic surgery.ti. or thoracoscopic surgery.ab. or thoracoscopy.ti. or thoracoscopy.ab. or thoracoscope.ti. or thoracoscope.ab. or thoracoscopes.ti. or thoracoscopes.ab. or video-assisted thoracic surgery.ti. or video-assisted thoracic surgery.ab. or video assisted thoracic surgery.ti. or video assisted thoracic surgery.ab. or minimally invasive thoracic surgery.ti. or minimally invasive thoracic surgery.ab. or minimally invasibility thoracic surgery.ti. or minimally invasibility thoracic surgery.ab. or minimally invasible thoracic surgery.ti. or minimally invasible thoracic surgery.ab. or minimally invasion thoracic surgery.ti. or minimally invasion thoracic surgery.ab. or minimally invasions thoracic surgery.ti. or minimally invasions thoracic surgery.ab. or minimally invasively thoracic surgery.ti. or minimally invasively thoracic surgery.ab. or minimally invasives thoracic surgery.ti. or minimally invasives thoracic surgery.ab. or minimally invasiveness thoracic surgery.ti. or minimally invasiveness thoracic surgery.ab. or minimally invasivity thoracic surgery.ti. or minimally invasivity thoracic surgery. ab. or minimally invasivity thoracic surgical procedures.ti. or minimally invasivity thoracic surgical procedures.ab. or minimally invasiveness thoracic surgical procedures.ti. or minimally invasiveness thoracic surgical procedures.ab. or minimally invasives proced surgical procedultit. or minimally ir masies thacic surgical procedures.ab. Or min thally invasive thoracic surgical

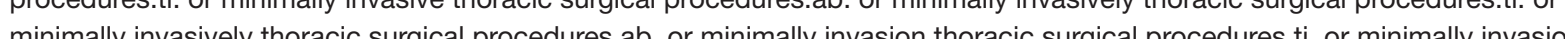
thoracic sure pros procedur or miniclic or minimally invasible thoracic surgical procedures.ti. or minimally invasible thoracic surgical procedures.ab.

\#2 chest tube ti. or chest tube ab. or chest tubes ti. or chest tubes ab or chest drain ti. or chest drain ab. or chest drainage. ti. or chest drainage ab. or chested drainage ti. or chested drainage ab. or chests drainage ti. or chests drainage ab. or thorax drainage ti. or thorax drainage ab or pleural drainage ti. or pleural drainage ab or pleura drainage ti. or pleura drainage ab. or thoraxes drainage.ti. or thoraxes drainage.ab.

\#3 (\#1 AND \#2)

Web of \#1 TS=(video-assisted thoracoscopic surgery) OR TS=(video assisted thoracoscopic surgery) OR TS=(thoracic surgery, video-assisted) OR TS=(VATS) OR TS=(thoracoscopic surgery) OR TS=(thoracoscopy) OR TS=(thoracoscope) OR $T S=$ (thoracoscopes) OR TS=(video-assisted thoracic surgery) OR TS=(video assisted thoracic surgery) OR TS=(minimally invasive thoracic surgery) OR TS=(minimally invasibility thoracic surgery) OR TS=(minimally invasible thoracic surgery) OR $T S=$ (minimally invasion thoracic surgery) $O R T S=$ (minimally invasions thoracic surgery) OR TS=(minimally invasively thoracic surgery) OR TS=(minimally invasives throcic surgery) OR TS=(minmally invasiveness Thoracic surgery) OR TS=(minimally surgical procactures) OR TS=(minimally invasives the codures) OR TS-(minim Tly in RTS-(minimally invasions thoracic surgical procedures) OR TS=(minimally invasility thoracic surgical procedures) OR TS=(nic) nimally invasible thoracic surgical procedures)

\#2 TS=(chest tube) OR TS=(chest tubes) OR TS=(chest drain) OR TS=(chest drainage) OR TS=(chests drainage) OR

TS=(chested drainage) OR TS $=$ (thorax drainage) OR TS=(thoraxes drainage) OR TS=(pleura drainage) OR TS=(pleural drainage) \#3 (\#1 AND \#2)

Cochrane \#1 (video-assisted thoracoscopic surgery):ti,ab,kw (Word variations have been searched) \#2 (video assisted thoracoscopic surgery):ti,ab,kw (Word variations have been searched) \#3 (thoracic surgery, video-assisted):ti, ab,kw (Word variations have been searched) \#4 (VATS):ti,ab,kw (Word variations have been searched)

\#5 (thoracoscopic surgery):ti,ab,kw (Word variations have been searched)

\#6 (thoracoscopy):ti,ab,kw (Word variations have been searched)

\#7 (thoracoscope):ti,ab,kw (Word variations have been searched)

(thored

(10 (video

H11 (minimally invasive thoracic surery) ti ab kw (Word variations have been searched)

\#12 (minimally invasibility thoracic surgery):ti,ab, kw (Word variations have been searched)

\#13 (minimally invasible thoracic surgeny):tiab, kw (Word variations have been searched)

\#14 (minimally invasion thoracic surgery).ti. ab. kw (Word variations have been searched)

\#15 (minimally invasions thoracic surgery):ti. ab. kw (Word variations have been searched)

\#16 (minimally invasively thoracic surgery):ti,ab, kw (Word variations have been searched)

\#17 (minimally invasives thoracic surgery):ti,ab,kw (Word variations have been searched)

\#18 (minimally invasiveness thoracic surgery):ti,ab, kw (Word variations have been searched)

\#19 (minimally invasivity thoracic surgery):ti, ab,kw (Word variations have been searched)

\#20 (minimally invasivity thoracic surgical procedures):ti,ab,kw (Word variations have been searched)

\#21 (minimally invasiveness thoracic surgical procedures):ti,ab,kw (Word variations have been searched)

\#22 (minimally invasives thoracic surgical procedures):ti,ab,kw (Word variations have been searched)

\#23 (minimally invasive thoracic surgical procedures):ti,ab, kw (Word variations have been searched) \#24 (minimally invasively thoracic surgical procedures):ti,ab,kw (Word variations have been searched) \#25 (minimally invasion thoracic surgical procedures):ti,ab,kw (Word variations have been searched) \#26 (minimally invasions thoracic surgical procedures):ti,ab, $\mathrm{kw}$ (Word variations have been searched) \#27 (minimally invasibility thoracic surgical procedures):ti,ab, kw (Word variations have been searched) \#28 (minimally invasible thoracic surgical procedures):ti,ab, kw (Word variations have been searched) \#29 (\#1 OR \#2 OR \#3 OR \#4 OR \#5 OR \#6 OR \#7 OR \#8 OR \#9 OR \#10 OR \#11 OR \#12 OR \#13 OR \#14 OR \#15 OR \#16 OR \#17 OR \#18 OR \#19 OR \#20 OR \#21 OR \#22 OR \#23 OR \#24 OR \#25 OR \#26 OR \#27 OR \#28)

\#30 (chest tube):ti,ab,kw (Word variations have been searched)

\#31 (chest tubes):ti,ab,kw (Word variations have been searched)

\#32 (chest drain):ti,ab,kw (Word variations have been searched)

\#33 (chest drainage):ti,ab,kw (Word variations have been searched)

\#34 (chests drainage):ti,ab,kw (Word variations have been searched) \#35 (chested drainage):ti,ab,kw (Word variations have been searched) \#36 (thorax drainage):ti,ab,kw (Word variations have been searched) \#37 (thoraxes drainage):ti,ab,kw (Word variations have been searched) \#38 (pleural drainage):ti,ab,kw (Word variations have been searched) 


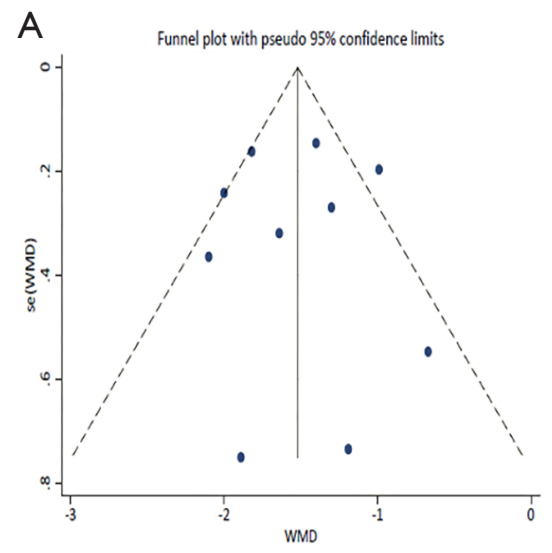

D

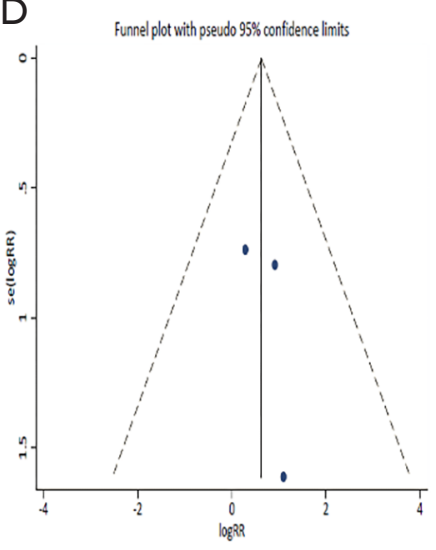

B

E

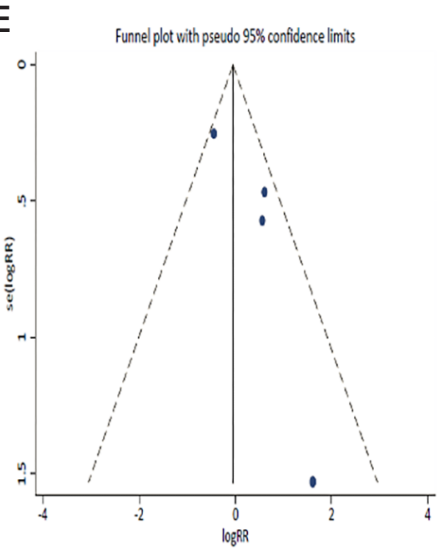

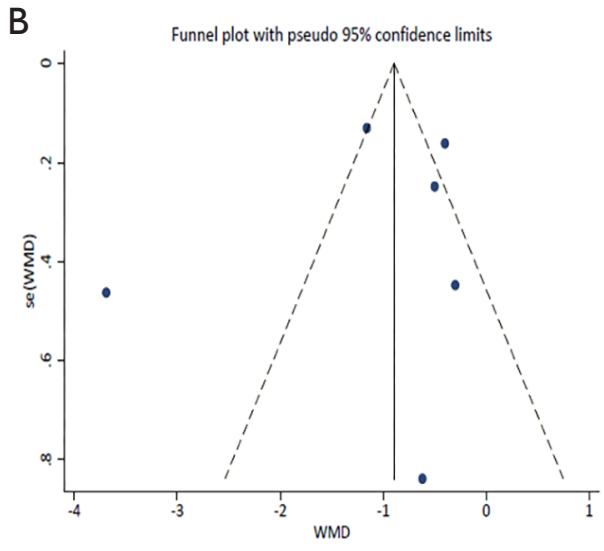

F

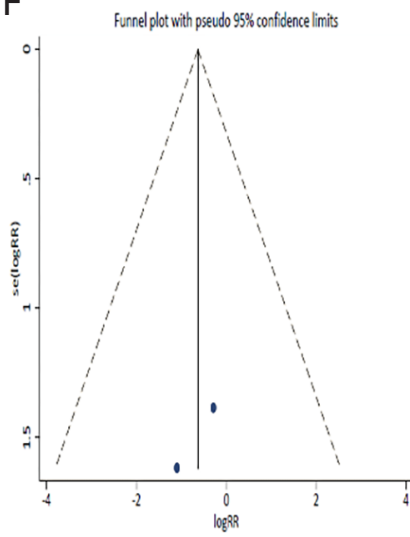

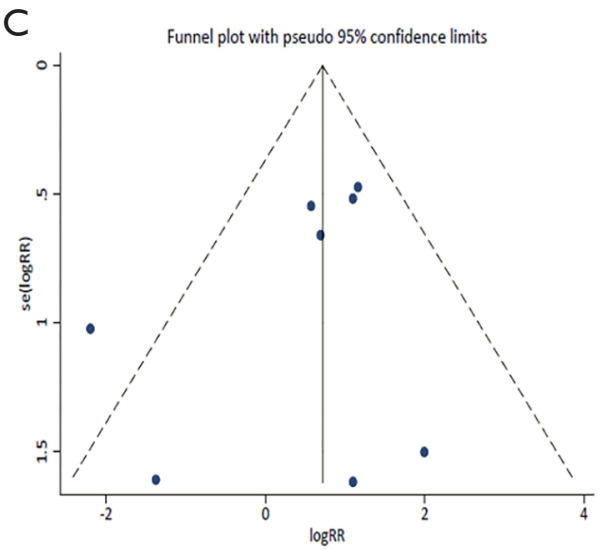

G

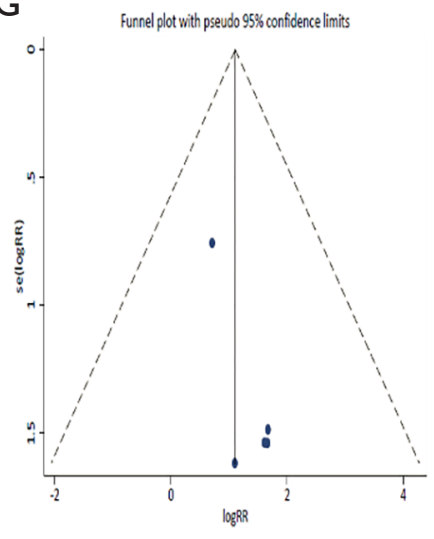

Figure S1 Funnel plot of (A) length of stay in hospital; (B) postoperative pain; (C) pneumothorax; (D) pleural effusion; (E) subcutaneous emphysema; (F) pneumonia; $(\mathrm{G})$ drain insertion or thoracentesis. 
Table S2 Criteria for intervention group and air leakage text of included studies

\begin{tabular}{|c|c|c|}
\hline Author & Criteria for intervention group & Air leakage test \\
\hline Luckraz et al. (19) & - No air leak was demonstrated & - Water sealing test \\
\hline Park et al. (32) & $\begin{array}{l}\text { - No previous ipsilateral thoracic surgery and secondary spontaneous pneumothorax } \\
\text { associated with underlying lung diseases such as pulmonary tuberculosis, empyema, cystic } \\
\text { fibrosis, and chronic obstructive pulmonary disease } \\
\text { - No air leak was demonstrated }\end{array}$ & - Water sealing test \\
\hline Lesser et al. (33) & $\begin{array}{l}\text { - No pulmonary nodules larger than } 3 \mathrm{~cm} \text { in diameter or location deeper to the visceral pleura } \\
\text { than the nodule diameter } \\
\text { - No more than one resection required } \\
\text { - No bullous emphysema or preoperative pleural effusion } \\
\text { - No intraoperative air fistula or diffuse pleural adhesions or bleeding or air leakage } \\
\text { - No injury of the visceral pleura, visible intraoperatively }\end{array}$ & $\begin{array}{l}\text { - Thoracoscopic } \\
\text { inspection } \\
\text { - Digital } \\
\text { measurement }\end{array}$ \\
\hline Liao et al. (34) & $\begin{array}{l}\text { - Nodule located within } 2 \mathrm{~cm} \text { of the visceral pleura and having a diameter }<2 \mathrm{~cm} \\
\text { - No moderate-to-severe obstructive or restrictive pulmonary diseases (GOLD guidelines: } \\
\text { presence of post-bronchodilator forced expiratory volume in one second (FEV1) }<80 \% \text { in } \\
\text { combination with a FEV1/forced vital capacity }(\mathrm{FVC})<70 \%) \\
\text { - No multiple wedge resections } \\
\text { - No air leaks following pulmonary resection } \\
\text { - No previous ipsilateral thoracic operation or pregnancy or lactation } \\
\text { - No other serious concomitant illnesses or medical conditions such as congestive heart failure, } \\
\text { bleeding tendency, liver cirrhosis, chronic renal disease, a condition requiring long-term use of } \\
\text { anticoagulants or steroids, or active infection requiring intravenous antibiotics }\end{array}$ & $\begin{array}{l}\text { - Water sealing test } \\
\text { - Vacuum ball }\end{array}$ \\
\hline $\begin{array}{l}\text { Steunenberg et al. } \\
\text { (27) }\end{array}$ & $\begin{array}{l}\text { - No air leak during the perioperative sealing test } \\
\text { - No bullous or emphysematous changes of the lung } \\
\text { - No dense pleural adhesions }\end{array}$ & - Water sealing test \\
\hline $\begin{array}{l}\text { Nakashima et al. } \\
\text { (28) }\end{array}$ & $\begin{array}{l}\text { - No air leaks during an intraoperative sealing test without additional suturing for endostaple } \\
\text { lines } \\
\text { - No bullous or emphysematous changes in the lung or dense pleural adhesion or preoperative } \\
\text { pleural effusion requiring chest drainage } \\
\text { - No high risk for postoperative bleeding } \\
\text { - For simultaneous bilateral resections, only the second side was considered for the study }\end{array}$ & - Water sealing \\
\hline Lu et al. (30) & $\begin{array}{l}\text { - Lesion located in the outer third of the lung field } \\
\text { - No bullous or emphysematous changes in the lung } \\
\text { - No air leaks, seen grossly or during a water sealing test } \\
\text { - No dense adhesion of pleura or oozing or accumulation of pleural effusion }\end{array}$ & - Water sealing test \\
\hline Liu et al. (38) & $\begin{array}{l}\text { - No tumour invasion to the great vessels or bilateral mediastinal surgery } \\
\text { - No unanticipated intraoperative bleeding or severe dense adhesion or accidental lung injuries } \\
\text { or air leaks } \\
\text { - No myasthenia gravis history } \\
\text { - No concomitant pulmonary wedge resections, pericardial window, or pleural biopsies }\end{array}$ & - Water sealing test \\
\hline Liu et al. (39) & $\begin{array}{l}\text { - Lesion of size } \leq 2 \mathrm{~cm} \\
\text { - No severe intrapleural adhesions observed during surgery } \\
\text { - No malignant or infectious pleural diseases or a history of thoracic surgery } \\
\text { - } \text { For anatomic pulmonary resection or } \geq 3 \text { individual unilateral wedge resections } \\
\text { under thoracoscopic vision and excluding those with haemothorax, pleural adhesions, lung } \\
\text { parenchymal disease and a history of thoracic surgery }\end{array}$ & $\begin{array}{l}\text { Digital } \\
\text { measurement/ } \\
\text { thoracoscopic } \\
\text { inspection and } \\
\text { water sealing test }\end{array}$ \\
\hline
\end{tabular}

